\title{
Réguler le dopage? Les failles de la gouvernance sportive
}

«L'affaire Puerto » comme illustration

\section{Bastien Soulé et Ludovic Lestrelin}

\section{OpenEdition}

\section{Journals}

Édition électronique

URL : http://journals.openedition.org/ress/1161

DOI : $10.4000 /$ ress. 1161

ISSN : 1663-4446

Éditeur

Librairie Droz

Édition imprimée

Date de publication : 15 juin 2012

Pagination : 127-159

ISBN : 978-2-600-01613-1

ISSN : 0048-8046

Référence électronique

Bastien Soulé et Ludovic Lestrelin, «Réguler le dopage ? Les failles de la gouvernance sportive »,

Revue européenne des sciences sociales [En ligne], 50-1 | 2012, mis en ligne le 15 juin 2015, consulté le 30 avril 2019. URL : http://journals.openedition.org/ress/1161 ; DOI : 10.4000/ress.1161 


\title{
RÉGULER LE DOPAGE? LES FAILLES DE LA GOUVERNANCE SPORTIVE "L'AFFAIRE PUERTO » COMME ILLUSTRATION
}

\begin{abstract}
BASTIEN SOULÉ \& LUDOVIC LESTRELIN
Université de Claude Bernard Lyon-I - Centre de recherche et d'innovation sur le sport \& Université de Caen Basse-Normandie - Centre d'étude sport et actions motrices bastien.soule@univ-lyonl.fr/ludovic.lestrelin@unicaen.fr
\end{abstract}

\begin{abstract}
Résumé. En 2006, une enquête de la Garde Civile espagnole, baptisée «opération Puerto», a conduit à l'identification d'un vaste réseau de dopage sanguin. Plus de cinq ans après, les sanctions prononcées à l'égard des protagonistes de cette affaire sont rares, et pour la plupart ténues, questionnant ainsi le processus de régulation propre à l'univers sportif. Nous postulons que c'est dans la nature particulière de l'organisation du sport, régi par une pluralité de pouvoirs de contrôle et de règles, que réside une clef de compréhension des difficultés qui marquent la lutte contre les infractions sportives. Le système régulateur est ainsi confronté à des difficultés de caractérisation des agissements qu'il est censé sanctionner et à la pluralité des sources juridiques et réglementaires. Dans le cas Puerto les rares condamnations ont été obtenues au prix de procédures lourdes, complexes, et devant faire preuve d'une ruse qui était jusqu'alors surtout l'apanage des dopeurs et des dopés.
\end{abstract}

Mots-clés: dopage, gouvernance, régulation, sport professionnel.

\begin{abstract}
In 2006, an investigation by the Spanish Guardia Civil led to the identification of a huge network practicing blood doping. Difficulties in terms of fight against doping of professional cyclists are analyzed using a systemic approach of governance. This article aims at enlightening the factors that made it impossible to impede the spreading of doping via auto-transfusion, as well as the attempts at restoring a balance among the professional cyclist elite. Through a qualitative inquiry, results show that timid attempts at gathering information prevent a full exposure of the affair and the punishment of its actors. The teleological priorities of many protagonists generate strategies preventing the identification of the network involved and the issuing of sanctions. Besides, we perceive the limits of a regulating system confronted with difficulties in characterizing the behaviors it is supposed to punish, with diverse legal and regulatory frameworks, and which is subject to an unwilling judicial authority.
\end{abstract}

Keywords: doping, regulation, governance, professional cycling. 
Mis au point dans les années 1970, le dopage par autotransfusion sanguine a été peu ou prou abandonné au début des années 1990, au profit de l'érythropoïétine (EPO) qui permet des effets similaires sur les performances sans nécessiter une logistique médicale aussi poussée. Depuis le début des années 2000, ce procédé ergogénique', proscrit par l’Agence Mondiale Antidopage (AMA) pendant et en dehors des compétitions, connaît toutefois un retour en grâce s'expliquant, d'une part, par les progrès réalisés en matière de détection de l'EPO et, d'autre part, par son caractère indécelable². À tel point qu'au printemps 2006, près de huit ans après les événements ayant marqué le Tour de France cycliste de 1998, un nouveau scandale sportif lié au dopage dans le cyclisme professionnel a éclaté en Espagne. Une enquête de la garde civile espagnole, baptisée «opération Puerto», a permis l'identification d’un réseau organisé se livrant à des prélèvements sanguins auprès de coureurs. Traité de manière à provoquer une concentration artificielle en globules rouges, le sang était conservé sous forme congelée, puis réinjecté aux cyclistes aux moments clés de la saison sportive afin d'améliorer leurs performances grâce au transport d'une quantité accrue d'oxygène vers les muscles.

Plutôt que de scandale sportif, il serait préférable de parler d'une «affaire Puerto». En effet, les scandales constituent des formes d'indignation partagée renforçant généralement la position de l'institution fédérale du fait d’un traitement essentiellement interne du problème. La mise au jour d'infractions se prolonge alors par la désignation de coupables puis leur condamnation, une manière de montrer l'action du mouvement sportif comme un «moyen de réaffirmer les valeurs du sport et de mobiliser le consensus autour de l'utopie sportive» (Lestrelin, Sallé, 2004, p. 220). A contrario, les affaires débordent le champ strictement sportif. Elles se caractérisent par le refus des contrevenants d'endosser le rôle de coupables, par l'intervention de la société dans son ensemble et

I C'est-à-dire permettant l'optimisation de la performance humaine. Voir De Léséleuc, Marcellini, 2005.

2 Les autotransfusions peuvent être indirectement mises en évidence par mesure du taux de globules rouges dans le sang. Cependant, il reste impossible de connaître avec précision le procédé à l'origine d'un fort taux d'hématocrite: prise d'EPO, séjour en altitude ou en caisson hyperbare, transfusion sanguine, etc. 
par la mobilisation des pouvoirs publics, juges, experts, médias, opinion, etc. (Duret, Trabal, 200I). «Pour le mouvement sportif, il s'agit [dès lors] d’affronter l'entrée dans l'arène de nombreux acteurs émanant d'institutions diverses, exigeant des preuves de nature différente et porteurs de principes de justice potentiellement contradictoires» (Demeslay, Trabal, 2007, p. I5 I). Tel semble être le cas ici.

Ainsi, ce n'est pas une volonté des institutions sportives mais une initiative publique qui est à l'origine des événements ${ }^{3}$. À ce titre, les conditions d'émergence de l'affaire Puerto ne sont pas sans rappeler celles de l'affaire Festina de 1998. Laction des forces de l'ordre espagnoles a été décisive dans la mise au jour du réseau organisé, tout comme l'avait été l'initiative des douaniers puis les investigations de la police judiciaire dans le cas français ${ }^{4}$. De telle sorte que l'affaire Puerto implique les tribunaux de droit commun. Comme dans l'affaire Festina encore, les instances sportives apparaissent quelque peu prises de court par le déroulement des événements, souvent placées en position de spectatrices. Au centre du réseau démantelé par l'enquête conduite par la police espagnole se trouve par ailleurs un médecin, Eufemiano Fuentes, rappelant une nouvelle fois que «les liens unissant les médecins, les sportifs de haut niveau et le dopage sont complexes et que des collusions structurent et organisent sa pratique depuis plusieurs décennies » (Sallé et al., 2006, p. 22)5. L’affaire Puerto vient en outre confirmer l'existence du dopage en tant que pratique planifiée, particulièrement développée dans le monde cycliste. Mais si, aujourd’hui, «parler de dopage dans le cyclisme n'est choquant pour personne» tant il y semble répandu et institutionnalisé (Lentillon-Kaestner, 2009, p. 519), l'ampleur du réseau mis au jour interpelle ici. En effet, l’affaire Puerto constitue probablement un «record»

3 À la différence d'autres cas de dopage révélés par des contrôles internes au mouvement sportif. Parmi les exemples les plus retentissants, on pense au sprinteur canadien Ben Johnson lors des Jeux olympiques de 1988.

4 C'est aussi l'action de la justice américaine qui a permis d'obtenir les aveux de l'athlète Marion Jones dans l'affaire dite Balco.

5 Sans verser dans une lecture du phénomène proche du fait divers, qui ferait de l'activité crapuleuse de «vrais-faux médecins» la source de tous les maux, rappelons l'ambiguïté des fondements de la médecine du sport, associant volonté de protection de la santé des sportifs et optimisation de leurs performances. Sur le rôle central des médecins dans l'histoire du dopage, voir notamment Waser, 2000; Basson et al., 200I ; Sallé, 2004. 
en matière de dopage organisé tant les sportifs professionnels impliqués sont nombreux. Qualifiée par la presse espagnole de «plus grande affaire de dopage de l'histoire», sa médiatisation a été très conséquente, d'autant plus que de nombreux rebondissements sont venus marquer le déroulement des événements.

Comment dès lors expliquer que cinq années après son déclenchement, l'affaire Puerto semble être «une montagne qui accouche d'une souris $»^{6}$ ? À ce jour, aucun coureur espagnol à l'exception d'Alejandro Valverde n’a été sanctionné. Et ce n'est que très récemment que le tribunal supérieur de justice de Madrid a annoncé que le Dr Fuentes ainsi que six autres personnes seront jugées, sortant quelque peu l'affaire de son enlisement ${ }^{7}$. Ainsi, le cas Puerto se pose comme un bon terrain d'observation pour questionner le processus de régulation des conflits et infractions (ici le dopage) propre à l'univers sportif. Ce faisant, nous aimerions suivre l'hypothèse selon laquelle c'est dans la nature particulière de l'organisation du sport, régi par une pluralité de pouvoirs de contrôle et de règles (règles spécifiques au sport, droit commun), que réside une clef de compréhension des difficultés qui marquent la lutte contre les infractions sportives. Dans le cas précis qui nous intéresse, notre regard porte sur la partie du processus située en aval des premières arrestations car elle représente un analyseur pertinent des priorités et des stratégies des acteurs faisant système autour de l'affaire. Source de contradictions et incertitudes, la diversité des groupes sociaux et des institutions interagissant dans la régulation du dopage (dont le champ de compétences déborde, pour certaines d'entre elles, largement le cadre national) rend problématique le prononcé de sanctions disciplinaires et/ou de droit commun. Préalablement à tout développement, il importe de situer notre étude sur les plans factuel et scientifique. Cela passe, d’une part, par un rappel synthétique du déroulement des événements et, d’autre part, par des précisions sur l’inscription disciplinaire et théorique du regard proposé.

6 Pour reprendre les termes prononcés par le procureur de la République dans son réquisitoire lors du procès d'une autre affaire de dopage dite «Cofidis », qui s'est déroulé au tribunal de grande instance de Nanterre du 6 au 10 novembre 2006. Ces propos sont cités dans Trabal et al., 2010, p. 99.

7 Nous détaillons plus loin les derniers développements de l'affaire. 


\section{CHRONOLOGIE ET PROTAGONISTES DE L'AFFAIRE}

C'est au sein d'un laboratoire clandestin installé dans un appartement madrilène du Dr Fuentes, figure centrale de l'affaire, qu'étaient réalisés puis stockés des prélèvements sanguins, entre autres pratiques ergogéniques : anabolisants, stéroïdes, hormones de croissance, doses d'EPO, poches de sang congelées et de plasma sanguin furent saisis. Auprès de ces poches figuraient des documents faisant état de procédés dopants à base de transfusions sanguines, administrés à de nombreux sportifs dont l'identité ne fut toutefois pas directement authentifiable du fait de l'usage combiné de numéros et de noms cryptés. Du matériel médical nécessaire au prélèvement, au traitement et à la conservation du sang a également été découvert.

Cinq personnes ont été arrêtées le 23 mai 2006. Outre le Dr Fuentes (ex-médecin de plusieurs équipes espagnoles: Kelme, Once...), il s'agit de Saiz (alors directeur sportif de l'équipe espagnole Liberty Seguros), de Labarta (directeur sportif adjoint de l'équipe Comunidad Valenciana), du Dr Merino (médecin chef du service d’hématologie de l'hôpital La Princesa de Madrid) et enfin de León («homme à tout faire» du Dr Fuentes). Seuls les deux médecins ont été placés en détention préventive, puis libérés après le paiement d’une caution de I20 ००० euro chacun.

S’appuyant sur le rapport judiciaire, le quotidien espagnol El Pais daté du 25 juin 2006 dévoile que 58 cyclistes professionnels, dont des coureurs de tout premier plan (Ullrich, Basso, Mancebo, Beloki, Contador, Sevilla), auraient eu recours aux «soins » du Dr Fuentes. Le I2 mars 2007, le juge d'instruction Serrano classe néanmoins l'affaire, au double motif qu'il n’existait pas de législation espagnole réprimant pénalement le dopage au moment des faits (une loi dans ce sens a été votée ultérieurement, le 2 novembre 2006), et que la dangerosité des produits et procédés en cause, non avérée ${ }^{8}$, rendait impossible la retenue des chefs d'incul-

8 Dans un rapport d'expertise daté du 22 décembre 2006, I'Institut National de Toxicologie de Madrid a estimé le niveau d'EPO détecté dans plusieurs poches de sang non risqué pour la santé (El Mundo, 18 février 2007). Le juge Serrano a de surcroît souligné les incertitudes scientifiques quant aux effets secondaires du recours à l'EPO. II a enfin minimisé les risques associés à la pratique des transfusions sanguines, notamment au regard de la dangerosité d'autres pratiques dopantes (As, II mars 2007). 
pation «atteinte à la santé publique» ou «mise en danger d'autrui». Le magistrat a par ailleurs mentionné quaucun des douze cyclistes auditionnés dans le cadre de la procédure n’a déclaré avoir subi de transfusion sanguine. Les "puertistes »" et les cinq personnes arrêtées le 23 mai 2006 bénéficièrent ainsi d’un non-lieu.

Le I4 mars 2007, le parquet de Madrid, représentant le Conseil Supérieur des Sports espagnol (CSS) ${ }^{\circ}$, bientôt rejoint par l'AMA, l’Union Cycliste Internationale (UCI) et la Fédération Espagnole de Cyclisme (FEC), a fait appel de cette décision, contestant l'innocuité des pratiques en vigueur chez le Dr Fuentes. Le I4 février 2008, le tribunal madrilène a repris l'instruction afin notamment de déterminer si le Dr Fuentes et ses présumés complices respectaient, au regard de la loi, les règles sanitaires relatives au prélèvement, au conditionnement, à la conservation et au transport du sang ${ }^{11}$. L'infraction de dopage ne pouvant être en l'espèce retenue, les débats relatifs à ce dernier ont été écartés.

Parallèlement, la garde civile a produit fin avril 2007 un second dossier Puerto, long de quelques 6000 pages, contre 500 pour la version initiale. La liste des «puertistes» s'allonge, passant de 58 à I07, et les indices permettant de préciser l'appartenance du sang contenu dans les poches saisies un an plus tôt se multiplient. Pourtant, le 29 septembre 2008, le juge Serrano prononce pour la seconde fois une ordonnance de non-lieu, motivant sa décision par l'absence d'éléments nouveaux permettant de caractériser les infractions évoquées ci-dessus. Le parquet et le CSS ont immédiatement interjeté un nouvel appel et le 12 janvier 2009, le tribunal provincial de Madrid a estimé que des indices d'un délit contre la santé publique pouvaient être retenus.

La réouverture du dossier a par conséquent été demandée: elle concerne les cinq personnes arrêtées le 23 mai 2006, ainsi que trois complices présumés (Belda, ex-manager de l'équipe Comunidad Valenciana; Cordova, ancien médecin de la formation Liberty Seguros; Yolanda Fuentes, la sœur du Dr Fuentes, médecin de l'équipe Comunidad Valenciana), qui encourent tous une peine maximale de deux ans

9 Selon le surnom donné aux coureurs nommés dans le rapport judiciaire établi par la garde civile.

10 Secrétariat d'État aux sports, organe central de l'administration publique du sport en Espagne.

II El Pais, 15 février 2008. La technique de l'autotransfusion est en effet porteuse de risques, notamment en cas de prélèvement en milieu non stérile ou de conditions hasardeuses de conservation. 
d'emprisonnement, ainsi que le retrait de leur carte professionnelle. À la fin du mois de novembre 20I , le tribunal supérieur de justice de Madrid annonce que sept personnes seront jugées pour «délit contre la santé publique», sans toutefois qu’une date soit précisée pour la tenue du procès. Outre Eufemiano Fuentes, les prévenus sont finalement les médecins José Luis Merino et Alfredo Cordova, la sœur du Dr Fuentes et les anciens directeurs sportifs d'équipes cyclistes Manolo Saiz, José Ignacio Labarta et Vicente Belda. À leur encontre, le parquet requiert une peine privative de liberté de deux ans et une interdiction d'exercice de leur profession de deux ans également, considérant qu’ils se sont rendus coupables de «pratiques visant à augmenter artificiellement le rendement de cyclistes $»^{12}$.

Cinq ans après les premières mises en cause, le processus judiciaire reste donc en suspens et les sanctions effectives sont pour le moment rares. «L'impression dominante est celle d'une escalade sans fin, d'une procédure interminable, sans gagnant définitif, sans bases juridiques incontestées » (Hourcade, 1996, p. I47) ${ }^{13}$. L’analyse de l'enchaînement des événements constitutifs de l'affaire met en évidence deux aspects : la pluralité des registres de recherche de la preuve, due notamment à l'absence de cadre législatif pénalisant le dopage en Espagne au moment des faits; le mécontentement généré par le faible nombre de prises permettant le prononcé de sanctions à l'encontre du «réseau Fuentes» et/ou des cyclistes mis en cause. La combinaison de ces deux éléments a conduit à des procédures alternatives de démonstration de la culpabilité de plusieurs parties prenantes. L’analyse proposée dans cet article entend étayer et affiner ce constat, afin notamment de mettre en évidence les difficultés qui caractérisent la régulation du monde sportif. Pour se révéler aussi complète que possible, l’analyse ne peut occulter les stratégies de plusieurs acteurs qui consistent, afin de se soustraire aux sanctions ou de protéger certains sportifs impliqués, à jouer de l'absence d'une règle de droit claire et/ou à s'immiscer dans les failles interstitielles générées par l'existence de registres normatifs concurrents.

12 Le Monde, 23 novembre 2011; El Mundo, 23 novembre 2011.

13 Si Michel Hourcade évoque ici l'affaire VA-OM de 1993 et le contrôle positif de l'athlète américain B. Reynolds au début des années 1990, il nous a semblé que ses mots s'appliquaient aussi au cas Puerto. 


\section{UNE ANALYSE SYSTÉMIQUE SOUS L'ANGLE DE LA GOUVERNANCE DU DOPAGE}

La retentissante affaire Festina de 1998 a entraîné une recrudescence des analyses sociologiques appréhendant le dopage via une approche en termes de système, c'est-à-dire comme «un ensemble d'interactions privilégiées entre des éléments, des acteurs ou des groupes d’acteurs et leurs produits » (Lugan, 1993). Selon un tel angle d'étude, le dopage est conçu comme produit systémique d'interactions entre de multiples acteurs ${ }^{14}$.

Les relations de pouvoir et la dynamique des configurations politiques au sein du cyclisme professionnel sont dès lors plus systématiquement étudiées (Stokvis, 2003; Stewart, Smith, 2008), tout comme les effets des fluctuations de l'environnement (Waser, 2000). La structuration et le fonctionnement du monde du cyclisme professionnel sont également abordés selon une perspective d’analyse systémique par B. Soulé et M. Bouhaouala (2008) qui montrent que le dopage est le fruit d'une causalité complexe. Il implique une diversité d'acteurs individuels et collectifs porteurs de projets hétérogènes, parfois concurrents, qui disposent de connaissances variées, entretiennent un rapport non uniforme aux règles et aux valeurs. Proposant de voir l'organisation du contrôle du dopage sportif comme un système complexe mettant aux prises particulièrement les dirigeants sportifs, les représentants des autorités publiques et le corps médical, L. Sallé et al. interprètent, quant à eux, «l'histoire et l'actualité du dopage non seulement comme le produit de l'évolution des relations qui régissent ces trois groupes identifiés, mais également comme le résultat de la domination ponctuelle d'un référentiel sur les deux autres» (2006, p. Io). Empruntée à la sociologie politique et particulièrement aux travaux qui s'intéressent aux politiques publiques (Muller, 2000), la notion de référentiel, appliquée au dopage, renvoie à un ensemble de pensées et de représentations produites

I4 Les travaux précurseurs réalisés par les sociologues de l'INSEP doivent cependant être signalés (Louveau et al., 1995). Ces chercheurs ont notamment montré que laisser le monopole du traitement du dopage aux responsables olympiques et fédéraux favorisait la généralisation des pratiques délictueuses: la position ambivalente de ces acteurs les pousse en effet à ne pas lutter efficacement contre le dopage ou à occulter certains faits gênants. Pour un état des lieux des travaux sociologiques menés sur le dopage, voir aussi Mignon, 2002. 
sur le phénomène qui conditionne et organise les actions des uns et des autres. En d'autres termes, toute intervention en matière de dopage « dépend, d’abord, de l'idée qu'ils s'en font et, ensuite, de leurs affrontements dans les mécanismes de régulation et gestion du phénomène » (Sallé et al., 2006, p. Io).

Au centre de ces approches systémiques, c'est en effet la question de la régulation du dopage qui est soulevée. Processus complexe qui renvoie aux moyens que se donnent les acteurs pour agir autour d’enjeux partagés et résoudre les problèmes et conflits posés par l'action collective (Reynaud, 1993), la régulation permet de pérenniser cette dernière, lui assure une forme de stabilité dans le temps. Elle est également ce qui permet de surmonter une crise. Les moyens qui fondent la régulation sont diversifiés, «de l'édiction de normes au recours à l'expertise, de l'allocation de ressources matérielles à la production discursive» (Dubois, 2009, p. 312). La justice est également un autre instrument.

En matière sportive, la régulation apparaît particulièrement complexe parce que le sport, marqué par ses origines locales et privées mais progressivement appréhendé comme un service public dans de nombreux pays européens, est régulé par un ensemble de décisions, d’actions et de pratiques diversifiées qui met en jeu des acteurs obéissant à des logiques différentes. Au gré des périodes, mouvement sportif et autorités étatiques nouent des alliances complexes, se posent en rivaux, relations «bipolaires» qui se résument finalement autour d'une «tension entre deux processus : l'autonomisation du monde sportif et ses organes, d'une part : les tentatives de mises sous tutelle pesant sur lui, d’autre part» (Defrance, 200I, p. 9) ${ }^{15}$. En France, par exemple, les années 1960 et 1970 sont celles de l’affirmation du sport comme entité bien établie, ayant une capacité de résistance à la pression venue du champ politique (Defrance, 1995). Si bien que les institutions qui forment le mouvement sportif (les instances fédérales en particulier) gagnent en souveraineté $^{16}$. Concentrant un ensemble de pouvoirs, elles régulent le sport selon leur propre logique, notamment via la production de normes sportives.

I5 D. Maliesky écrit également que les relations entre le monde du sport, particulièrement les instances olympiques, et les autorités publiques sont passées «par toute une série de configurations allant de l'éloignement à la soumission, sans omettre une hostilité déclarée» (2001, p.47).

16 D'une manière générale, le sport déroge à la souveraineté de l'État de deux façons : par les 
En effet, l'autonomisation du sport va de pair avec «la constitution d'un corpus de règles de plus en plus précises et contraignantes, par le fait de spécialistes qui légifèrent en dehors du droit commun» (Hourcade, 1996, p. I48). «Principe organisateur » (Reynaud, 1993, p. 4), la règle est couplée à l'existence de moyens de contrôle et de sanction. Le monde du sport génère donc ses propres organes, normes, décisions, son propre circuit de traitement des problèmes, autant d'instruments de régulation qui visent à assurer sa pérennité et garantir son autonomie. «Le sport, comme système, présente ainsi tous les éléments constitutifs d'un ordre [...]: à la fois ordonnancement, c'est-à-dire "agencement d'éléments disparates et hétérogènes en un ensemble cohérent et intelligible", et commandement, "manifestation d'autorité” propre à réaliser et maintenir la cohésion et l'unité du système» (Simon, 200Ia, p. 99).

Comme tout ordre, l'ordre sportif «est nécessairement confronté à son environnement [...], aux autres ordres existants» (Simon, 200Ia, p. I0o). La tendance est particulièrement accentuée depuis les années 1980 alors que le professionnalisme se développe et que corruption, affairisme, violences et dopage portent le sport régulièrement à la rubrique des faits divers. Le mouvement d'expansion des règles étatiques au détriment des règles privées place de plus en plus le «sport à l'épreuve du droit commun» (Miège, 200I) 17. Judiciarisation du sport, règles spécifiques régissant le domaine sportif complétées ou contredites par les principes généraux du droit national, prise en compte obligatoire du droit communau$\operatorname{taire}^{18} \ldots$ « Ces dernières années ont vu, tant au niveau communautaire que dans

règles qu'il élabore et par la possibilité offerte aux participants d'exercer en toute légitimité une violence physique contrôlée. Voir Hourcade, 1996. Sur les rapports tumultueux entre sphère privée (le sport) et sphère publique (l'État), voir aussi pour le cas français G. Simon (1990) et pour la dimension européenne J.-L. Chappelet (20I0).

17 C'est particulièrement vrai pour le cas du dopage. L'intrusion des pouvoirs publics s'explique par des enjeux d'affirmation et de garantie de l'ordre public. L. Sallé (200I, p. I63) écrit ainsi: «la circulation de produits illicites dans des structures subventionnées par l'Etat pose la question du rapport que les sportifs entretiennent aux normes et aux règles établies [...]. À ce titre, le dopage peut être considéré comme une remise en cause de l'ordre public car il est non seulement contraire aux règles sportives, mais parce qu'il est de surcroît interdit par la loi (au regard du trafic qu'il génère) et dangereux pour la santé ».

18 On pense notamment à l'arrêt pris en 1995 par la Cour de justice des communautés européennes (aujourd'hui renommée Cour de justice de l'Union européenne), dit «Bosman» qui marque en la matière un tournant. Sur la rencontre du sport et du droit communautaire, voir entre autres Husting, De Waele, 2001. 
l'espace du droit national, une accélération des tensions, des frottements entre la norme étatique et les règlements du sport » (Besnier, 20ı, p. 43). Dans la mesure où la logique concurrentielle et commerciale tend également à s'imposer, la norme sportive est par ailleurs remise en cause par un autre «acteur», également producteur de «lois»: le marché (Simon, 200Ib). De telle sorte que la régulation du domaine sportif répond aujourd'hui à un système au sein duquel les règles, plurielles, sont rarement en conformité, parfois se situant même dans une franche opposition $^{19}$. Prêtant à toutes les confusions, ce système est encore complexifié par le morcellement des acteurs qui composent l'ordre sportif (institutions olympiques, fédérations nationales et internationales), par la pluralité des acteurs publics (États et leurs divers services, Union européenne, organismes internationaux) qui investissent l'arène sportive et par la multiplication des échelles spatiales (nationales, internationales, communautaires) qu'induit la mondialisation du sport ${ }^{20}$.

La lutte antidopage est concernée par ces constats. Bien qu'étant destinées à «régler des problèmes » et à mieux contrer le phénomène, les règles en la matière n'en entraînent pas moins plusieurs effets pervers. Comme le rappellent J. Demeslay et P. Trabal, «selon que l'on qualifie un sportif de citoyen (et donc justiciable selon les lois de son pays), ou de compétiteur (relevant donc des règlements sportifs de la fédération), ses droits et devoirs sont potentiellement variables, voire contradictoires » (2007, p. 150). La prohibition du dopage est, par ailleurs, décrite comme étant peu efficace, puisqu'elle inciterait à produire et utiliser des produits mal connus, de fait pas encore détectables (Caulkins, Reuter, 2005), voire à user d’agents masquants pouvant se révéler dangereux (Bird, Wagner, 1997 ; Voy, 1991 ; Stewart, Smith, 2008). Le recours à l'automédication de coureurs n'osant plus solliciter de conseillers médicaux, avec son lot de dosages approximatifs (Waddington, 200I) ou encore l'incitation indirecte au dopage sanguin que constitue l'acceptation tacite, par l'UCI, de seuils d'hématocrite anormalement élevés (Soulé, Bouhaouala, 2008) sont d’autres exemples.

19 C'est l'objet même de l'ouvrage dirigé par J.-C. Basson (200I). Voir en particulier la première partie.

20 Pour ce qui est du dopage, cet état de fait pose la question de l'harmonisation de la lutte. C'est le rôle dévolu à l'AMA, créée en 1999. Le code mondial antidopage en est l'outil principal. Sur ce point, voir Demeslay, Trabal, 2007. 
La complexité décrite ici impose dès lors au chercheur de respecter certaines conditions pour mener son étude. Analyser le système de régulation du dopage, objectif assigné à cet article, suppose de dresser prioritairement la «carte des parties prenantes » de l'affaire de manière à éclaircir tout d’abord le propos (c'est l'objet de la figure I, présentée ci-dessous) ${ }^{21}$. Sur un plan temporel, nous entendons nous focaliser précisément sur le réseau d’acteurs interagissant à partir de la mise en évidence de l'existence de procédés proscrits. En conséquence, les aspects liés à la détection des dits procédés, à leur développement au sein du cyclisme professionnel, tout comme la question de leur prévention, ne sont pas abordés. La partie du processus régulateur qui nous intéresse ici est celle qui a été déclenchée par l'intervention des forces de l'ordre espagnoles, et qui était censée se prolonger par des sanctions disciplinaires et/ou de droit commun.

Figure I: Les principales parties prenantes de l'affaire Puerto

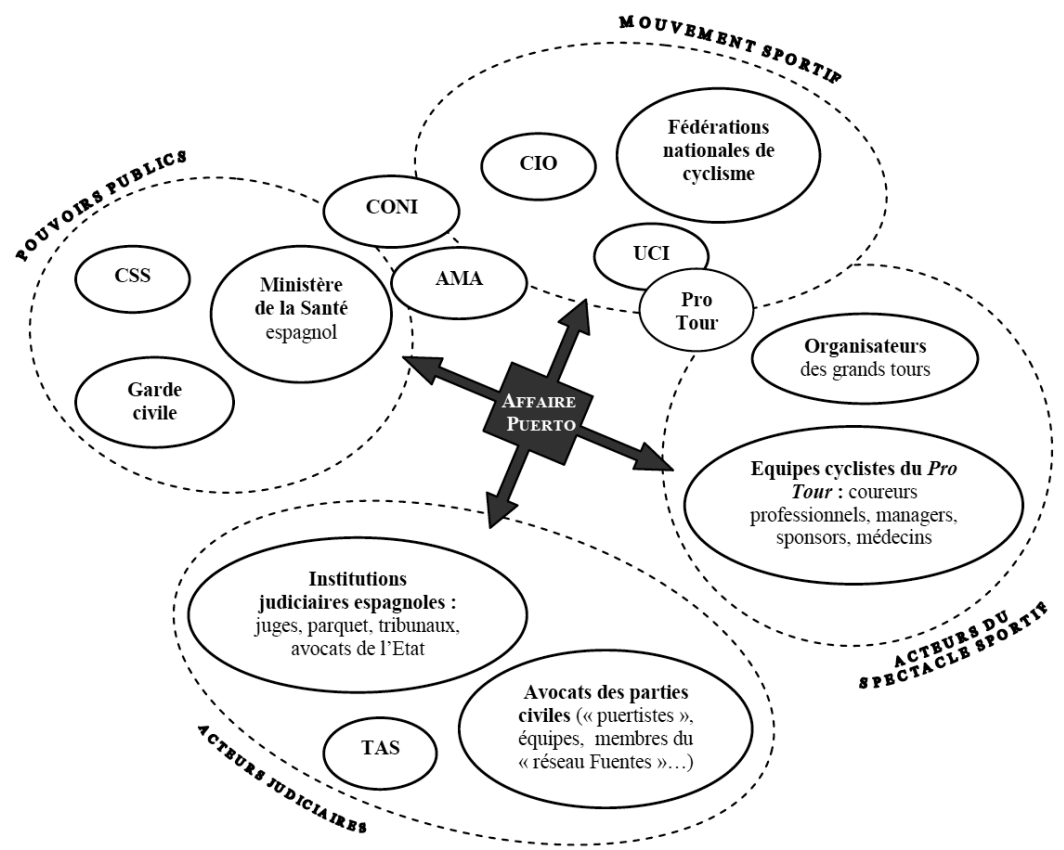

21 À cette étape de la démonstration, il s'agit donc d'ordonner sous forme synthétique la présentation des différents protagonistes identifiés dans le point, qui ouvre cet article, consacré à la chronologie de l'affaire. 
L'étude de ce système complexe renvoie finalement aux débats autour de la gouvernance. Comme l'affirment P. Trabal et al. (20ı, p. 7), «le cas du dopage est emblématique de la redéfinition des frontières de l'État et des interrogations suscitées autour de la gouvernance ». Cette notion polysémique (qui connaît un succès certain) est généralement utilisée pour marquer une évolution, celle du « dépassement des politiques antérieures qui ont montré leurs limites et leurs insuffisances » (Gaudin, 2002, p. 9) pour faire face à l'exigence de régulation dans des contextes de plus en plus instables, mouvants et complexes. En ce sens, elle interroge traditionnellement la restructuration de l'action publique et l'abandon du modèle classique de politiques menées à titre principal par un État centralisé sur des secteurs bien délimités. Dans le champ sportif, l’usage relativement récent de la notion de gouvernance traduit les difficultés des instances sportives à gérer les «dérives» du sport, la nécessaire intervention d'autres instances régulatrices et l'importance d'une coopération plus systématique avec les acteurs publics. Évoquer la gouvernance sportive revient alors à constater la multiplication croissante des acteurs et des espaces par et dans lesquels se développent et s'exercent les politiques sportives.

L'intérêt de la notion de gouvernance, pris non sous l'angle de la recommandation de «bonnes pratiques » de gouvernement mais selon son acception systémique, tient à ce qu'elle vise à rendre compte des situations d'interdépendance et des régulations inter-organisationnelles en situation complexe: incertitudes de l'environnement, multiplicité des acteurs (publics, associatifs, marchands) obéissant à des intérêts divergents et pluralité des échelles de coordination (Bayle, Chantelat, 2007). Nous envisageons donc la gouvernance à travers «les problèmes de coordination que posent une telle diversification des acteurs, de scènes et des formes d'interaction » (Lascoumes, Le Galès, 2007, p. I09-IIo). Le lien entre gouvernance et dopage a déjà été opéré à l'échelon français par L. Sallé (2004) qui rend compte des acteurs prenant en charge le phénomène et qui montre combien ces derniers font système, à travers des formes de coordination multi-niveaux et multi-acteurs, révélatrices de rapports de force construits à partir de visions plurielles du dopage et d'intérêts contradictoires. 
Ainsi abordée, l'affaire Puerto se pose comme un terrain d'observation privilégié pour restituer l'environnement incertain, les problèmes de coordination entre acteurs, la précarité du «partage des prérogatives [et] de la répartition des rôles et des missions» (Trabal et al., 20ıо, p. 188) entre pouvoirs publics et instances sportives qui caractérisent la gouvernance contemporaine du dopage. Génératrice de «désordre» (Gaudin, 2002), celle-ci laisse opérer de puissants facteurs de dérégulation qui offrent non seulement de nombreuses opportunités d’atteintes à l'ordre public (Basson, 200I) mais rendent également délicats le prononcé de sanctions et la résolution des conflits.

\section{MÉTHODE}

Nous nous sommes livrés à une analyse des articles de presse consacrés à l'affaire Puerto, de mai 2006 à la seconde réouverture du dossier par la justice espagnole à l'été 2009. S'imposer cette butée temporelle a permis de ne pas engager une course-poursuite contre l'actualité journalistique, incompatible avec le rythme de la recherche (Marchetti, 2002). Nous avons toutefois continué à assurer une veille de manière à saisir les derniers développements de l'affaire. Le matériau recueilli est constitué de 80 articles émanant de 18 titres européens. L’Equipe, Le Monde, As, El Pais, Le Nouvel Observateur et Libération sont les plus mobilisés. Viennent ensuite Le Figaro, puis un ensemble de titres ponctuellement mis à contribution : Le Parisien, La Gazzetta dello Sport, L’Humanité, Marca, La Tribune, Le Temps, Le Soir, El Mundo, Le Journal du Dimanche, La Libre Belgique et Der Spiegel. Les informations collectées l'ont été à deux desseins :

- Reconstituer aussi fidèlement que possible, sur un plan factuel, la trame des événements, à partir des traces que constituent les déclarations officielles, procédures et décisions de justice, aveux, etc. Cette tâche est compliquée par la présence de déclarations contradictoires, de démentis et de retournements de situation faisant immanquablement subsister des zones d'ombre.

- Permettre, par l'intermédiaire de ces comptes rendus, interviews et prises de position, l'identification des facteurs interdépendants ayant contribué au relatif statu quo observé. Le repérage des désaccords et controverses passibles d'une lecture stratégique est notamment visé : jeux autour de la pluralité et de la légitimité relative des référentiels juridiques; signes d'une volonté d’opacification 
ou de révélation de faits centraux de l'affaire; tentatives de mise en cause ou de sauvegarde, a contrario, de la responsabilité des coureurs impliqués ; etc.

Le premier type d'exploitation des données vise une contextualisation, aussi précise que possible, de l'étude. Le second est plus clairement inscrit dans la problématique énoncée plus haut; il implique la sélection, au sein du corpus constitué, des aspects permettant une mise en perspective de l'affaire, et par là même le dépassement du simple récit. Il est à noter que contrairement à ce qu'ont pu observer C. Delmas et S. Fleuriel (2002) à propos de la manière dont les médias relatent les affaires de dopage, plusieurs articles parus dans la presse témoignent d'une certaine mise à distance critique par rapport aux sources officielles et au traitement institutionnel de l'affaire. La principale limite de cette étude réside néanmoins dans le caractère secondaire des données exploitées, fruits d'interprétations journalistiques pour la plupart. Pour l'heure, seul ce type de méta-analyse permet de contourner l'impossibilité d'accès au dossier judiciaire, à moins de s'interdire d'aborder l'affaire sous un angle scientifique.

\section{RÉSULTATS}

\section{I. LES LIMITES DE L'AUTORÉGULATION AU SEIN DU MONDE DU CYCLISME PROFESSIONNEL}

L’affaire Puerto a été déclenchée par l'intervention d’acteurs publics s'étant substitués aux autorités sportives. C'est un premier aveu d'impuissance de ces dernières, censées identifier et sanctionner le dopage; démonstration est ainsi faite de leur incapacité manifeste à organiser efficacement la lutte, voire de leur manque de volonté d’agir. Suite aux premières révélations, les frémissements au sein du cyclisme professionnel furent cependant tout sauf anodins. Plusieurs sponsors d'équipes comptant dans leurs rangs des «puertistes» ont mis un terme à leur soutien ${ }^{22}$. En interne, des managers ont pris la décision de suspendre certains coureurs avant, en fonction des cas, de les licencier ou de les réintégrer. Certains cyclistes et formations se sont par ailleurs vu retirer leur invitation au

22 Liberty, assureur américain, justifie cette décision par le «tort causé à son nom et au nom du cyclisme par l'arrestation de Manolo Saiz» (dépêche AFP, 27 mai 2006). Pas moins de quinze «puertistes» étaient sous contrat avec cette équipe. 
départ d'épreuves majeures ${ }^{23}$, précipitant leur déclin. Cinq équipes professionnelles ont du reste été dissoutes à la suite de cette affaire, entraînant nombre de coureurs vers une fin de carrière anticipée ${ }^{24}$. Ces répercussions internes (à ce jour les plus concrètes dans le cadre de l'affaire Puerto) semblent motivées par des craintes d'atteinte à l'image de ces partenaires, équipes professionnelles et organisateurs d'événements. Elles sont liées à des stratégies de survie économique propres à certains acteurs cherchant à pérenniser l'activité qui les fait vivre, que l'on voit se dessiner plus fréquemment depuis le début des années 2000 dans le cyclisme professionnel (Brissonneau et al., 2009; Fincoeur, 2009). Changements de posture, inflexion des discours, évolution des pratiques... Autant de transformations augurant a priori, si l'on veut être optimiste, d'une possible rupture avec la «norme du dopage », banalisée et culturellement associée au cyclisme dans les années 1980 et 1990 (Lentillon-Kaestner, 2009)25.

Dans le même temps, des accords solennels, augurant de capacités d'autorégulation du monde du cyclisme professionnel, ont reçu l'adhésion de plusieurs acteurs majeurs le composant. Ils n’ont toutefois pas résisté à la prégnance des intérêts particuliers ainsi qu’à la vive concurrence économique et sportive régnant au sein du cyclisme professionnel. Ainsi, le code éthique proposé par l’UCI, signé par l’intégralité des équipes du Pro Tour après les premières révélations de mai 2006, consistait en un engagement moral (donc sans contrainte

23 À l'image de l'équipe Comunidad Valenciana (au sein de laquelle officiait Labarta) et des coureurs Ullrich et Basso pour l'édition 2006 du Tour de France (Libération, 16 février 2008).

24 Dans le détail, un an après l'opération, 30 «puertistes» avaient trouvé refuge en Elite 2 (division inférieure); 46 avaient mis un terme à leur carrière; 8 avaient changé de discipline (VTT, piste); enfin, une petite dizaine espérait encore trouver une formation.

25 Ces stratégies, induites par l'intrusion d'acteurs publics et la menace de sanctions devant la loi, semblent confirmer plus largement que pour ce qui concerne le sport, une pratique telle que le dopage «a d'autant plus de chances d'être dénoncée et qualifiée de délictueuse qu'elle menace l'autonomie de l'ordre dans lequel elle a cours» (Slimani, 20I0, p. 668). P. Chantelat (200I) a aussi montré que la stratégie des instances sportives face à la tricherie et aux « dérives» consiste régulièrement à désigner les corrompus et les tricheurs (volontiers qualifiés d'éléments «malsains») puis à les condamner en les expulsant du monde sportif. Outre des profits symboliques et performatifs, elle procure des avantages concrets: maintien d'une autonomie et de pouvoirs, réanimation d'un idéal fondateur mythifié, celui d'un sport vu comme un univers pur et propre par essence. 
juridique) à licencier toute personne formellement convaincue de dopage, à suspendre de toute compétition les coureurs sous le coup d'une enquête, et à ne pas s'attacher les services de ces coureurs avant que ne soit rendue une décision de justice ${ }^{26}$. Devant les opportunités d'embauche représentées par les premiers licenciements, plusieurs équipes sont passées outre cette convention, engageant à moindre coût des «puertistes » fraîchement congédiés. Ces agissements ont partiellement ruiné les velléités de régulation interne de l’affaire.

\subsection{LE «LAISSER-FAIRE»}

\section{ET LA MISE SUR AGENDA TARDIVE DU DOPAGE EN ESPAGNE} Une absence de prise pour la justice de droit commun

C’est la diffusion alarmante du dopage en Espagne, inhérente au laxisme officiellement reconnu par le gouvernement Zapatero, qui encouragea, début 2005, la mise en place d'une politique de «tolérance zéro $»^{27}$, traduite en 2006 par l'adoption d’une législation pénale. Vue sous cet angle, l’affaire Puerto constitue la résultante d'une tolérance tacite du dopage en Espagne, lequel, y compris dans sa modalité transfusionnelle, était organisé de longue date dans le cyclisme professionnel, au vu et au su de plusieurs acteurs institutionnels.

Une des limites imposées à l'identification des «tricheurs» réside dans la nécessité de faire entrer les procédures de recherche de preuves dans un cadre répréhensible. Or, s'il serait exagéré d’affirmer que ce dernier faisait défaut en Espagne au moment des faits ${ }^{28}$, la législation réprimant pénalement la production, l'usage et la distribution de produits dopants (avec des peines allant jusqu'à 2 ans d’emprisonnement) n’a été adoptée qu’a posteriori, en novembre 2006. tionale des Groupes Cyclistes Professionnels, sous la forme d'un engagement moral.

27 Libération, 17 février 2005.

28 Le titre 8 de la loi du 15 octobre 1990 stipule que «le CSS est en charge de promouvoir toutes les mesures de prévention, de contrôle et de répression du dopage» (Miège, 2000, p. 7l). Une ordonnance de 1996 précise les procédures d'homologation et de contrôle des laboratoires, alors qu'un décret de la même année établit le régime des sanctions, et qu'une résolution du CSS du II février 1997 dresse la liste des procédés et substances prohibés. Contrairement à celle adoptée en novembre 2006, cette loi ne prévoyait pas de sanction pénale, et laissait à l'écart les pourvoyeurs. 
Ainsi, les faits constatés par la garde civile ne tombent pas sous le coup de la nouvelle législation (principe de la légalité de la peine).

Alors que les connaissances scientifiques actuelles permettent de comparer les poches de sang saisies par la garde civile avec l’acide désoxyribonucléique $(\mathrm{ADN})$ des coureurs mis en cause, confirmant ou infirmant, avec un risque d'erreur quasi-nul, leur implication, une telle procédure serait infondée. Seules deux démarches de ce type ont été entreprises: l'une, imposée par la justice allemande, a conduit à confondre le coureur Ullrich (voir infra) ; l'autre, entreprise par le Comité Olympique Italien (CONI), prouve que Valverde, meilleur cycliste aux classements UCI Pro Tour 2006 et 2008, a recouru aux services du Dr Fuentes ${ }^{29}$. Tous les «puertistes » à qui il fut demandé de recourir à de telles comparaisons pour prouver leur innocence ont refusé de s'y soumettre. Ce n'est que par le biais de prélèvements sanguins réalisés à l'occasion de contrôles anti-dopage ultérieurs que ces tests ont pu être réalisés.

Dès la fin des années 1990, d’autres pays européens (parmi lesquels la France ou l'Italie) ont fait des choix différents, que traduit l'adoption de textes législatifs altérant en profondeur les rapports de force: les acteurs sportifs ont alors perdu du pouvoir au bénéfice des acteurs publics ${ }^{30}$. Cette redistribution des cartes a notamment été provoquée par la faillite, l'incapacité, voire l'absence de réelle volonté des autorités sportives de lutter efficacement contre le dopage (Sallé et al., 2006). L'affaire Puerto illustre sur ce plan un décalage temporel contribuant à rendre délicate, sinon impossible, la prise de sanctions au regard du droit commun, et limitant, par voie de conséquence, les opportunités de sanction sportive (voir infra).

29 Le Nouvel Observateur, Ier avril 2009; L'Equipe, 24 février 2009.

30 Après l'affaire Festina de 1998 et la «découverte» que le dopage sévissait à grande échelle dans le sport, C. Brissonneau explique que «le dopage fait l'objet d'une reprise en main législative par de nombreux États ouest-européens et anglo-saxons, dont la France avec la loi du 23 mars 1999» (2010, p.454). Sur le processus de production de cette loi, dite «Buffet» (du nom de la ministre des sports alors en fonction), voir Sallé, 2004. 


\subsection{LES ENTRAVES JUDICIAIRES}

\section{À LA PRODUCTION ET À LA TRANSMISSION DE PREUVES}

Les éléments abordés ci-dessus suffisent-ils à expliquer les réticences constatées en matière de recueil et de production d'informations relatives à l'affaire, ainsi que la communication parcellaire, sélective et tardive des éléments sensibles du dossier? Ne fournissent-ils pas surtout un argument juridiquement fondé, permettant d'occulter les pratiques dévoilées et/ou de minimiser l'étendue du réseau mis au jour? En tout état de cause, les pièces à conviction se sont faites rares, rendant problématique la prise de sanctions, y compris disciplinaires. Constat d'autant plus surprenant que les saisies des forces de l'ordre furent massives, tout comme fut conséquente la somme de conversations téléphoniques, photographies et enregistrements vidéo collectée par les enquêteurs.

L’avocat de l'État a ouvertement déploré que le juge Serrano n’ait pas exploité les données disponibles sur les ordinateurs saisis par la garde civile. On sait par ailleurs de source journalistique ${ }^{31}$ que le Dr Fuentes consignait les informations relatives à ses «patients» sur des fiches conservées dans sa résidence Canarienne. Cette dernière n’a pas été perquisitionnée, alors que l’on avait tout loisir de le faire.

De plus, l'intégralité de la centaine de poches de sang saisies n’a pas été analysée. Seules huit d'entre elles ont fait l'objet d'une recherche d'EPO, positive dans tous les cas. Les autres n'ont pas été traitées, le laboratoire antidopage de Barcelone en charge de l'analyse ayant suspendu ses activités suite au non paiement des premières expertises ${ }^{32}$. Enfin, le juge n’a entendu que I2 coureurs parmi les 107 «puertistes», au motif qu’aucun ne s'était plaint de problèmes de santé.

Au-delà de cette production a minima d'informations sur l'affaire et ses protagonistes, la transmission d'éléments du dossier aux différentes instances sportives susceptibles de prononcer des sanctions disciplinaires posa également problème. Un mois après l'opération, le président de l’UCI mettait déjà en doute

31 Le Monde, 24 mars 2007.

32 Le Monde, 7 décembre 2006. La poursuite des analyses, ordonnée par le juge pour mieux cerner l'appartenance du sang saisi et détecter d'éventuels traitements, est coûteuse. Le laboratoire en charge de les réaliser l'a donc conditionnée au paiement de la somme de 25000 dollars déjà due (El Pais, 16 janvier 2007). 
la volonté de collaborer des autorités espagnoles. Fin 2006, il a officiellement déploré le manque de coopération de la justice espagnole ${ }^{33}$, qui avait préalablement refusé de lui transmettre les éléments constitutifs du dossier. Le juge Serrano avait alors invoqué l'impossibilité d’autoriser l'utilisation de tels documents à des fins de sanction disciplinaire. En réaction, le 23 avril 2007, l'UCI, par l'intermédiaire de son président, l'AMA et le CIO ont lancé un appel urgent au Ministre des Sports espagnol afin que l'affaire Puerto fasse l'objet d'investigations en profondeur ${ }^{34}$. La requête restera vaine. Près de trois ans après le déclenchement de l'opération, le président de l'AMA a une nouvelle fois tenté de faire pression sur les magistrats afin d'exiger la transmission des preuves rassemblées pendant l'enquête ${ }^{35}$. Au même moment, en mai 2009, la Fédération Espagnole de Cyclisme a essuyé un refus de la part du juge d'instruction lorsqu'elle a sollicité l'accès au dossier, l’argument invoqué étant devenu l'absence d'élément permettant de prouver une mise en danger de la santé publique.

Cette démarche pour le moins timorée de collecte d'informations, combinée à l'absence d'échange transparent de données entre institutions régulatrices, empêche de faire toute la lumière sur l'affaire et, dans une certaine mesure, de sanctionner les acteurs impliqués. Une minoration de l'ampleur du réseau identifié est induite, alors que le flou persiste quant à sa composition réelle. Du côté des instances sportives, le mécontentement généré par les deux classements sans suite de l'affaire se double d'un ressentiment grandissant à l'encontre des instances judiciaires, voire des autorités espagnoles, à qui il est explicitement reproché de ne pas tout mettre en ouvre pour lever le voile d’opacité entourant les agissements repérés.

33 Par l'intermédiaire d'un courrier adressé au Ministre des Sports espagnol (Eurosport, 26 juin 2007).

34 Le Parisien, 24 avril 2007.

35 Le Nouvel Observateur, 28 février 2009. 


\subsection{LA PROTECTION OFFERTE AUX «PUERTISTES » ESPAGNOLS}

Si cette affaire est d'importance, c'est, entre autres aspects, du fait du nombre de cyclistes concernés. Paradoxalement, c'est peut-être l'élément qui explique la légèreté des sanctions prononcées : se montrer plus sévère conduirait en effet à écarter un grand nombre de coureurs, parmi lesquels de nombreux espagnols et/ou ténors du circuit. Au-delà, un changement de perspective s'impose afin de cerner des enjeux dépassant le seul cyclisme professionnel. La liste des tennismen et footballeurs professionnels qui auraient aussi bénéficié des soins du Dr Fuentes n’a jamais été divulguée ${ }^{36}$. Les cyclistes sont, en effet, les seuls «puertistes » à avoir vu leur identité divulguée dans la presse.

Pat Mc Quaid (président de l'UCI) a plusieurs fois sous-entendu que les espagnols cherchaient à dissimuler quelque chose ${ }^{37}$. Du reste, le recours en annulation déposé le 22 mars 2007 par l'avocat de l'État mentionne que le classement de l'affaire est intervenu «de façon précipitée et surprenante » ${ }^{38}$. Ne cherche-t-on pas ainsi à restreindre l'ampleur réelle de cette embarrassante affaire? Ne s'agit-il pas in fine d'éviter de faire de l'ombre au sport de haut niveau espagnol, en réussite exceptionnelle en cette fin de décennie $2000^{39}$ ? Il y a plus de io ans, Basson et al. (200I, p. 29I) observaient déjà qu’en tant qu'«élément constitutif de la culture et de l’identité nationale, le sport [semble jouir] d'une sorte d'impunité qui voit le dopage, sinon favorisé, du moins banalisé : ne sert-il pas la bonne cause ? » Les assertions catégoriques et préci-

36 Ce tri des informations serait destiné à ne pas rendre publique l'implication des dits sportifs (Le Monde, 7 décembre 2006). II est écrit dans cet article que plusieurs équipes espagnoles de football (parmi lesquelles le Real Madrid et le FC Barcelone) sont liées au Dr Fuentes. Cette affirmation vaudra au quotidien une condamnation à 300000 euro d'amende, suite à une plainte déposée par le Real Madrid. Le Journal du Dimanche du 2 février 2006 met en cause, outre des footballeurs, le tennisman Rafael Nadal.

37 Le Temps, 26 février 2008.

38 Le Monde, 24 mars 2007.

39 Jaime Lissavetsky, Secrétaire d'État et président du CSS, qualifia 2008 d'«année magique du sport espagnol»: titres internationaux obtenus en cyclisme (Tours de France, d'Italie et d'Espagne, Jeux Olympiques), tennis (Coupe Davis, Wimbledon, Jeux Olympiques) et football (Championnat d'Europe). Le chef du gouvernement, Jose Luis Zapatero, avança pour sa part: «Nous comptons parmi les meilleurs pays au monde. Grâce à vos succès, il est facile d'être dans le G8 du sport» (El Pais, 23 novembre 2008). 
pitées d'éminents représentants des pouvoirs publics espagnols, cherchant à minorer l'étendue du réseau de sportifs en lien avec le Dr Fuentes ${ }^{40}$, renforcent le caractère plausible de cette explication.

Moins pondéré, un spécialiste de la lutte antidopage explique que «l’Espagne est le pays qui, vis-à-vis du dopage, pose le plus de problèmes en Europe $»^{41}$. Selon les présidents de l'UCI et de la FEC, l'Espagne serait le «paradis du dopage ${{ }^{42}}^{2}$, ce que confirme le médecin d'une équipe professionnelle : «il est admis, sauf pour les autorités espagnoles, que l'Espagne est la plaque tournante du dopage européen, pour tous les sports $»^{43}$. La pression exercée sur les coureurs supposément impliqués est bien plus forte en Allemagne ou en Italie, alors que la présomption d'innocence permet à de nombreux cyclistes espagnols de bénéficier d'une relative clémence ${ }^{44}$. Situation d'autant plus surprenante que l'Espagne fait partie, en Europe, des pays se caractérisant par une intervention poussée de l'État et un certain centralisme gestionnaire dans le domaine du sport, au motif que sa promotion et son développement sont envisagés comme étant d’intérêt général (Miège, 2000).

À ce jour, la justice espagnole n’a autorisé aucun acteur du mouvement sportif à utiliser les éléments du dossier judiciaire à des fins de sanction disciplinaire, rendant quasi-intouchables les coureurs et managers suspectés. Seuls quatre coureurs ont fait l'objet de suspensions: Basso (deux ans) et Scarponi (I8 mois), deux italiens sanctionnés par la FCI après être passés aux aveux

40 Le 4 juillet 2006, Jaime Lissavetsky se voulait rassurant: «Aucun footballeur ni joueur de tennis n'est impliqué» (Libération, 7 décembre 2006). Destinée à couper court aux affirmations selon lesquelles des footballeurs et tennismen espagnols de premier plan seraient clients du DrFuentes, cette dénégation fut néanmoins contestée par ce dernier dès le lendemain.

4l Le Temps, 26 février 2008 (informateur souhaitant conserver l'anonymat).

42 Libération, 16 février 2008. Le président de l'UCI a réitéré cette affirmation récemment, déclarant: «L'Espagne a un problème avec le dopage», Le Monde, 8 janvier 20 II. La mise au jour en décembre 2010 d'un vaste réseau de dopage organisé dans l'athlétisme espagnol, surnommée «affaire Galgo», semble confirmer ces propos.

43 Gérard Guillaume, médecin de l'équipe la Française des Jeux (L'Humanité, 19 juillet 2008).

44 As, 10 mai 2007. 
devant le $\mathrm{CONI}^{45}$; Valverde, coureur espagnol interdit de course pendant deux ans sur le sol italien ${ }^{46}$; Jaschke, suspendu un an par la Fédération Autrichienne de Cyclisme, également suite à sa reconnaissance des faits ${ }^{47}$.

\subsection{DES ORDRES JURIDIQUES CONTRADICTOIRES, PROPICES À UN JEU SUR LES RÈGLES}

Le nombre d'organisations ayant a priori la possibilité de constater des infractions, de prendre des sanctions et/ou de prononcer des arbitrages est conséquent: UCI, fédérations nationales, AMA, Tribunal Arbitral du Sport (TAS), institutions judiciaires de chaque pays, Ministères ou Secrétariats d'Etat au sport, comités nationaux olympiques, $\mathrm{CIO}$, etc. ${ }^{4}{ }^{8}$ Ces diverses institutions, s'appuyant sur des textes réglementaires propres, et, pour certaines, des listes de produits et procédés interdits, revendiquent une légitimité dans la lutte contre le dopage. Il en résulte une situation de pluralisme juridique ${ }^{49}$ (Rocher, 1989), ou juxtaposition de corps de normes pluriels (étatiques, non étatiques, privés...). Immanquablement porteuse de commandements simultanés incompatibles ${ }^{50}$ (Carbonnier, 2004),

45 Concernant Basso, le CONI a brandi la menace d'un test $A D N$, rendu possible après I) qu'il se soit procuré des échantillons sanguins auprès du laboratoire barcelonais en charge des analyses; 2) qu'il ait réalisé un prélèvement sanguin auprès de Basso lors d'un contrôle antidopage inopiné. Le vainqueur du Tour d'Italie 2006 et challenger d'Armstrong sur le Tour de France 2005 est dès lors passé aux aveux, Scarponi l'imitant peu après.

46 L'Équipe, I4 mai 2009. Lourde de conséquence, cette interdiction mit Valverde dans l'impossibilité de prendre le départ du Tour de France 2009, dont la 16 étape intégrait un passage par le Val d'Aoste, en Italie (Le Figaro, 18 mai 2009 et Le Monde, 15 juin 2009). Le coureur espagnol a fait appel de cette sanction devant le TAS, mais l'arbitrage de ce dernier n'a pu être rendu avant le départ du Tour (Le Monde, 23 juin 2009).

47 La Tribune, 19 septembre 2007, Le Monde, 28 juillet 2007 et Der Spiegel, 30 juin 2007.

4812 des 17 communautés autonomes espagnoles disposent de surcroît d'une compétence en matière sportive et de leur propre loi sur le sport. Le CSS est censé coordonner leur action, notamment à propos du sport de haut niveau, de la lutte contre le dopage ou des faits de violence (Miège, 2000).

49 Plus largement, le pluralisme juridique est constitutif du droit du sport, en France comme dans les autres pays européens: cette polysystémie résulte de l'internationalisation du droit et des instances sportives, qui diluerait selon F. Latty (2007) la souveraineté de chaque droit national.

50 L'UCl, pourtant prompte à dénoncer le manque de coopération de la justice et des autorités espagnoles, est elle-même dans l'impossibilité de fournir des échantillons sanguins en sa possession pour permettre la réalisation de comparaisons d'ADN. Elle se trouve prise au piège 
elle engendre une dilution des mises en cause, des situations de blocage ainsi que d'importants délais entre procédures respectives ${ }^{51}$. Ces difficultés de coordination sont propices à un jeu qui contribue à dévoiler les stratégies des protagonistes (repli sur les textes offrant une protection, usage défensif des règles de procédure, etc.). Les rebondissements autour du cas de Valverde (voir cas I, ci-dessous) sont à ce titre riches d'enseignements.

\section{Cas I : le cas Valverde ou la stratégie perdante du «bras de fer»}

Le 29 août 2007, au regard des informations portées à sa connaissance par le biais du second dossier Puerto, I'UCl a interdit Valverde de participation aux championnats du monde. Le 7 septembre, la FEC a refusé toute mesure disciplinaire à l'encontre de Valverde, annonçant du même coup son intention de le retenir dans sa sélection. Le secrétaire des sports espagnol a apporté son soutien au coureur, mettant l'accent sur l'absence de nouveaux éléments prouvant son implication. Cette prise de position de l'État espagnol justifiera un courrier adressé au secrétaire des sports par le président de l'UCl, soulignant l'existence de preuves accablantes de la culpabilité de Valverde, et déplorant que l'Espagne ne fasse pas preuve de transparence.

Estimant ne pas disposer d'éléments suffisants pour interdire Valverde de course, le TAS l'a autorisé à participer aux mondiaux ${ }^{52}$. L'AMA et l'UCl ont vainement demandé au juge espagnol d'avoir accès à une poche de sang portant un code supposé désigner Valverde, afin de pratiquer un test ADN. Le TAS a alors demandé la production de la poche, mais le juge Serrano a refusé, au motif que le statut d'association privée du TAS excluait toute possibilité de transmission des preuves. Ce refus a occasionné le II juillet 2008 un appel conjoint de l'UCl et de l'AMA pour l'application de la décision du TAS. En juillet 2008, le TAS a donné à la justice espagnole un délai de six mois pour fournir cette poche de sang ${ }^{53}$.

Valverde est au cœur d'un autre imbroglio. Lors d'un bref passage du Tour de France 2008 sur le sol italien, le CONI a soumis ce coureur à un prélèvement sanguin. Une comparaison ADN avec un échantillon de sang saisi lors de l'opération Puerto a permis de confirmer son appartenance à Valverde. Le tribunal antido-

de son propre règlement, qui interdit la communication de tels échantillons à des fins autres que scientifiques (Dépêche Associated Press, 10 août 2006).

5I La FEC a ainsi attendu de se voir notifier la décision de classer l'affaire sans suite avant de réagir: «quand nous l'aurons, nous prendrons une décision. Cela dépendra de la décision du parquet, qui a la possibilité d'interjeter appel de la décision du juge» (Libération, 13 mars 2007).

52 Décision du TAS (2007/O//38I RFEC \& Valverde c/ UCl) consultable sur son site officiel.

53 Marca, 14 avril 2008; L'Équipe, II juillet 2008. 
page du CONI, soutenu par l' $U \mathrm{Cl}$, a dès lors prononcé la sanction évoquée supra (voir sous-partie 4.4.), pour violation du code de l'AMA.

Le coureur espagnol a quant à lui porté plainte contre le CONI, estimant qu'il avait transgressé l'interdiction, signifiée par la justice espagnole, d'utiliser des éléments du dossier Puerto ${ }^{54}$. Son équipe (Caisse d'Épargne) a taxé le CONI d'organisme incompétent et qualifié la procédure d'irrégulière. Pour sa part, le tribunal supérieur de justice de Madrid a déclaré nulle la procédure de recueil de preuves du CONI, au double motif que I) celui-ci, ne représentant pas la justice italienne, n'était pas en droit de réclamer en Espagne des échantillons sanguins susceptibles de confondre Valverde ${ }^{55}$; 2) les preuves collectées lors de l'enquête espagnole ne sauraient être utilisées dans le cadre d'une autre affaire ${ }^{56}$. Le président du Comité Olympique Espagnol a de même souligné l'innocence de Valverde. La FEC complète ce front de soutien au coureur espagnol, jugeant qu'elle est «la seule compétente pour imposer les sanctions disciplinaires relatives aux cas de dopage $»^{57}$. On confine enfin à l'affaire d'État lorsque le Secrétaire d'État aux Sports espagnol rappelle qu'il revient à la justice espagnole de juger le cas de son ressortissant.

Selon le procureur italien en charge de cette affaire, lors de l'audience de Valverde, ses avocats n’ont pas répondu aux accusations, se contentant d'expliquer que les autorités italiennes n’avaient pas légitimité à agir dans cette affaire.

On perçoit aisément les limites d'un système régulateur confronté à des difficultés de caractérisation des agissements qu'il est censé sanctionner, à la pluralité des sources légales et réglementaires, et de surcroît assujetti à une autorité judiciaire peu volontariste. Les rares condamnations obtenues le sont au prix de procédures lourdes, complexes, devant faire preuve d'une ruse qui était jusqu'alors surtout l'apanage des dopeurs et des dopés. Leur lenteur offre en outre un répit aux acteurs mis en cause, dont la majorité officie encore au sein du cyclisme professionnel.

54 Le Nouvel Observateur, 6 mai 2009.

55 L'Equipe, 18 février 2009.

56 Le Nouvel Observateur, Ier avril 2009.

57 Le Figaro, 18 mai 2009. 


\subsection{LE NÉCESSAIRE RECOURS À DES REGISTRES ALTERNATIFS DE MISE EN ÉVIDENCE DES DÉLITS}

Les sanctions prononcées à l'encontre de Valverde sont le fruit de la ténacité du CONI qui s'était résolu, dès janvier 2008, à entamer une procédure alternative, annonçant son souhait d'entendre les personnes impliquées ${ }^{58}$ et n'excluant pas de prononcer des sanctions disciplinaires ${ }^{59}$. Une autre procédure, d'autant plus remarquable qu'elle s'émancipe, par la force des choses, des règles disciplinaires ou étatiques relatives au dopage, a permis de confondre Ullrich (voir cas 2, ci-dessous).

\section{Cas 2: les circonvolutions ayant permis la réalisation d'un test ADN auprès d'Ullrich}

Ne disposant pas de loi sur le dopage au moment des faits, la justice allemande a privilégié une démarche alternative de démonstration de la culpabilité d'Ullrich, à travers une plainte pour fraude et escroquerie vis-à-vis du public, des sponsors et de ses coéquipiers. Ce coureur a effectivement eu recours à des procédés dopants alors qu'il avait signé un document auprès de son équipe T-Mobile dans lequel il excluait de recourir à des substances prohibées. II a ainsi perçu des salaires qui ne lui auraient pas été versés si son employeur en avait eu vent. Ce concept juridique n'avait encore jamais été appliqué à un cas de dopage présuméc0. Suite au test ADN prouvant la culpabilité du coureur allemand (le sang contenu dans neuf poches saisies chez le Dr Fuentes lui appartenait bel et bien), le parquet de Bonn lui a proposé un marché: reconnaître sa culpabilité à travers le paiement d'une somme de 250000 euros, reversée à des œuvres ainsi qu'au trésor public, en échange d'un classement sans suite de l'enquête. Le coureur s'est acquitté de cette somme pour obtenir l'abandon de la procédure. En vertu de cet accord, Ullrich n'est plus considéré coupable d'aucun délit.

D’autres changements de registre des poursuites ont été entrepris. En Espagne, la mise en danger d'autrui et l'atteinte à la santé publique, tour à tour invoquées pour tenter d’activer le processus judiciaire, n’ont cependant pas rendu possible la désignation de responsables, du fait de l’absence de mise en

58 L'Equipe et Le Figaro, 10 janvier 2008. Constatant l'impossibilité de miser sur l'aide du système judiciaire espagnol, le CONI abandonnera néanmoins son entreprise (dépêche AFP, 26 juin 2008).

59 Depuis août 2007, la règlementation anti-dopage entrée en vigueur en Italie permet d'interdire aux athlètes étrangers dont la responsabilité dans une affaire de dopage est établie de concourir sur le territoire italien (L'Équipe, 14 janvier 2008).

60 Le Nouvel Observateur, 10 mars 2008. 
évidence d'effets néfastes sur la santé des cyclistes. Quand bien même la dangerosité des transfusions sanguines est reconnue, et qu'il est avéré que plusieurs acteurs se sont bel et bien livrés à ce procédé dopant ${ }^{61}$, il reste difficile de démontrer qu'une personne a été exposée à un risque tant qu'elle demeure en bonne santé, ce qui est le cas des «puertistes». La seconde réouverture du dossier évoquée en début d’article est motivée par deux hypothèses de violation du code pénal ${ }^{62}$ : le Dr Fuentes et son réseau ont-ils respecté les règles de conditionnement prévues par la législation sur les médicaments (respect de la chaîne du froid concernant les poches de sang; conditions de conservation des produits comme l'EPO) ? La pratique d’actes médicaux (prélèvement et/ou injection de sang) a-t-elle été le fait de personnes habilitées ? Finalement, à la fin du mois de novembre 20I r, le tribunal supérieur de justice de Madrid annonce que sept personnes seront jugées pour «délit contre la santé publique». Le dopage ne figure plus au cœur de l'arsenal juridique mobilisé. Seuls quatre médecins et trois directeurs sportifs restent sur la sellette, les coureurs étant simplement entendus, le cas échéant, comme témoins.

En ce sens, les derniers développements de l'affaire Puerto semblent s'inscrire dans un mouvement plus général, se caractérisant, en termes de régulation, par la prédominance de la perception sanitaire et médicale du phénomène. Cette vision consacre la préservation de la santé comme principe de légitimation de l'action des pouvoirs publics en matière de dopage ${ }^{63}$.

61 Plusieurs journaux ont publié des extraits d'un rapport du juge Serrano, affirmant le 12 mars 2007 que bien qu'il soit convaincu que des coureurs ont eu recours au dopage par autotransfusion, ces agissements sont insuffisamment étayés pour caractériser l'infraction de mise en danger de la santé publique.

62 Libération, 16 février 2008.

63 En France, la loi de 1999 sur la protection de la santé des sportifs marque un tournant. Cette période vient rompre avec une époque, celle par exemple de la première loi antidopage de 1965, pendant laquelle l'argument moral était au principe de l'action de l'État en faveur du sport, pensé comme un service d'intérêt général. Voir Le Noé, 2000. 


\section{CONCLUSION}

Plus de cinq ans après les faits, l'AMA et l'UCI n'en finissent pas de souligner leur frustration, ainsi que la perte de crédibilité que génère ce feuilleton judiciaire «à rallonge $»^{64}$. Évoquer à propos de l'affaire Puerto une crise de gouvernance du dopage est a priori tentant. Plusieurs éléments militent pour l'usage du terme de crise : nombre conséquent et grandissant de parties prenantes; présence de vives controverses alimentées par la conflictualité des enjeux; caractère spectaculaire du traitement médiatique de l’affaire; étiolement de la légitimité des acteurs clés du système de la lutte antidopage ${ }^{65}$. Par ailleurs, la difficulté éprouvée pour appréhender l’affaire dans toutes ses dimensions et la cohabitation cacophonique d'acteurs judiciaires, étatiques et sportifs plaident bel et bien pour la désignation de cette affaire en tant que crise.

Toutefois, les contextes de crise, par définition limités dans le temps, sont généralement assortis de tentatives de retour à l'état d’origine. Ce dernier est-il souhaitable, alors que le cas d'espèce se caractérise précisément par une absence d'état initial stable, une résilience pour le moins limitée des acteurs, voire une certaine duplicité de certaines parties prenantes? Il semble préférable d'estimer que l'affaire Puerto révèle les failles de la gouvernance sportive, qui rendent aujourd'hui très délicates la résolution des conflits et des problèmes, ainsi que le prononcé de sanctions. Quelle que soit l'appréciation que l'on peut porter sur cette situation, il serait injuste d'en tirer la conclusion selon laquelle la lutte antidopage s'avère une entreprise impossible. Bien qu'erratique, la régulation du dopage, soumise à de multiples difficultés et résistances, a fait l'objet en Espagne d'une mise en politique qui permet d'envisager les événements décrits comme une période propice à un changement accéléré, à la clarification des responsabilités et prérogatives de chaque partie prenante et à une éventuelle redistribution des cartes ${ }^{66}$.

\section{L'Équipe, 2 décembre 2009.}

65 Force est de constater que l'AMA, dont la vocation est d'harmoniser les différents dispositifs légaux et sportifs en matière de dopage, n'a guère assumé son rôle.

66 Selon P. Lascoumes et P. Le Galès (2007, p. 80), «il y a mise en politique lorsqu'une question est déployée en dehors de son espace de formulation originel et devient incontournable tant pour les médias que pour les responsables politiques et administratifs ». 
Analyser le dopage revient en fait à observer la «territorialisation» de l'action publique en matière sportive en train de se faire, «en ce sens que la prise en compte du phénomène [du dopage] par les différentes autorités de régulation politique et administrative pose le problème de la délimitation d'un espace de compétences - sous la double acception du terme - où puissent se développer des solutions appuyées sur une connaissance fine de ce phénomène travaillé par la globalisation qui n’épargne pas le secteur sportif » (Trabal et al., 2oı, p. 6). Et c'est en se livrant à une sociologie politique de l'action publique que l'on pourra penser ces nouvelles configurations en cours entre les acteurs publics et la société sportive, «entre une (ou des) autorité(s) publique(s) démocratiquement légitimée(s) et les formes de mobilisation des acteurs sociaux qui s'efforcent d'organiser et de promouvoir leurs intérêts symboliques autant que matériels » (Lascoumes, Le Galès, 2007, p. II2).

NdA. Les auteurs remercient Frédérique Roux, juriste, Maître de Conférences à l'Université Lyon I, pour ses conseils avisés lors de la phase de rédaction de cet article.

\section{BIBLIOGRAPHIE}

BASSON Jean-Charles (dir.), 200I, Sport et ordre public, Paris, La Documentation française-IHESI.

-, FÉRET Jérôme, LOUVEAU Catherine, WASER Anne-Marie, 200I, «Conclusion: dopage sportif et recomposition des pouvoirs de contrôle. Jalons pour une recherche en cours», in Jean-Charles Basson (dir.), Sport et ordre public, Paris, La Documentation française-IHESI, p. 289-294.

BAYLE Emmanuel, CHANTELAT Pascal, 2007, La Gouvernance des organisations sportives, Paris, L'Harmattan.

BESNIER Frédéric, 2010, «Organisations sportives: l'union fait la force», Jurisport, I02, p. 42-45.

BIRD Edward J., WAGNER Gert G., 1997, «Sport as a common property resource», Journal of Conflict Resolution, 4I-6, p. 749-766. 
BRISSONNEAU Christophe, 20I0, «Dopage», in Michaël Attali, Jean Saint-Martin (dir.), Dictionnaire culturel du sport, Paris, Armand Colin, p.453-455.

-, DEFRANCE Jacques, FINCOEUR Bertrand, LENTILLON-KAESTNER Vanessa, OHL Fabien, (2009), Carrière sportive et socialisation secondaire en cyclisme sur route: les cas de la Belgique, la France et la Suisse, Rapport de recherche pour l'Agence Mondiale Antidopage.

CARBONNIER Jean, 2004, Sociologie juridique, Paris, PUF.

CAULKINS Jonathan P., REUTER Peter, 2005, «Re-defining the goals of national drug policy: recommendation from a working group», American Journal of Public Health, 85-8, p. 1059-1063.

CHANTELAT Pascal, 200I, «Sport, enjeux économiques et corruption: crépuscule ou renaissance de l'utopie sportive», in Jean-Charles Basson (dir.), Sport et ordre public, Paris, La Documentation française-IHESI, p. 25I-269.

CHAPPELET Jean-Loup, 2010, L'Autonomie du sport en Europe, Strasbourg, Conseil de l'Europe.

DEFRANCE Jacques, 1995, «L'autonomie du champ sportif. 1890-1970», Sociologie et Sociétés, 27-I, p. |5-31.

-, 200I, «Introduction: histoire du sport, régulations sociales et contrôle public. Entre autonomisation et mise sous tutelle», in Jean-Charles Basson (dir.), Sport et ordre public, Paris, La Documentation française-IHESI, p. 9-19.

DE LÉSÉLEUC Eric, MARCELLINI Anne, 2005, «Légitimité vs illégitimité du dopage chez les sportifs de haut niveau. Comment se définissent les limites du non acceptable?», Revue internationale des sciences du sport et de l'éducation physique, 70-4, p. 33-47.

DELMAS Corinne, FLEURIEL Sébastien, 2002, «Une vision enchantée de la vie professionnelle: l'éthique à l'épreuve des conditions du travail sportif et journalistique», Les Cahiers du journalisme, II, p.34-47.

DEMESLAY Julie, TRABAL Patrick, 2007, «De quelques contraintes du processus d'harmonisation des politiques antidopage», Terrains et Travaux, 12, p. 138-162. 
DUBOIS Vincent, 2009, «L'action publique», in Antonin Cohen, Bernard Lacroix, Philippe Riutort (dir.), Nouveau manuel de science politique, Paris, La Découverte, p. 311-325.

DURET Pascal, TRABAL Patrick, 200I, Le Sport et ses affaires. Une sociologie de la justice de l'épreuve sportive, Paris, Métailié.

FINCOEUR Bertrand, 2009, «Lutte antidopage et cyclisme à deux vitesses: évolution du rapport au dopage chez les cyclistes belges depuis l'affaire Festina», Revue internationale de criminologie et de police technique et scientifique, 3, p. 207-220.

GAUDIN Jean-Pierre, 2002, Pourquoi la gouvernance?, Paris, Presses de Sciences Po. HOURCADE Michel, 1996, «Le sport et l'État de droit: approche sociologique», Droit et Société, 32, p. 141-155.

HUSTING Alexandre, DE WAELE Jean-Michel, 200I, Sport et Union européenne, Bruxelles, éditions de l'Université de Bruxelles.

LASCOUMES Pierre, LE GALÈS Patrick, 2007, Sociologie de l'action publique, Paris, Armand Colin.

LATTY Franck, 2007, La lex sportiva. Recherche sur le droit transnational, Leiden, Martinus Nijhoff Publishers.

LE NOÉ Olivier, 2000, «Comment le dopage devint l'affaire des seuls sportifs», in Françoise Siri (dir.), La Fièvre du dopage, Paris, Autrement, p. 77-92.

LENTILLON-KAESTNER Vanessa, 2009, «Appropriation progressive de la culture du dopage dans le cyclisme », Déviance et Société, 33-4, p. 519-54I.

LESTRELIN Ludovic, SALLÉ Loïc, (2004), «Le sport et ses valeurs: mobilisation des acteurs et élaboration d'un consensus», in Florence Carpentier (dir.), Le Sport est-il éducatif? Les valeurs: leur constitution, leur transmission. Approches historiques, sociologiques, philosophiques, Rouen, Presses universitaires de Rouen, p. 219-229.

LOUVEAU Catherine, AUGUSTINI Muriel, DURET Pascal, IRLINGER Paul, MARCELLINI Anne, 1995, Dopage et performance sportive. Analyse d'une pratique prohibée, Paris, INSEP. 
LUGAN Jean-Claude, 1993, La Systémique sociale, Paris, PUF.

MALIESKY Dominique, 200I, «Le Comité international olympique: une diplomatie parallèle », in Jean-Charles Basson (dir.), Sport et ordre public, Paris, La Documentation française-IHESI, p. 9-19.

MARCHETTI Dominique, 2002, «Sociologie de la production de l'information», Cahiers de la Recherche sur l'Éducation et les Savoirs, I, p. 17-32.

MIÈGE Colin, 2009 [2000], Les Organisations sportives et l'Europe, Paris, Insep éditions. -, 200I, «Le sport à l'épreuve du droit commun: la fin d'une exception?», in Jean-Charles Basson (dir.), Sport et ordre public, Paris, La Documentation française-IHESI, p. 23-36.

MIGNON Patrick, 2002, Le Dopage: état des lieux sociologique, Paris, CESAMES, 10.

MULLER Pierre, 2000, «L'analyse cognitive des politiques publiques: vers une sociologie politique de l'action publique», Revue française de science politique, 50-2, p. 189-207.

REYNAUD Jean-Daniel, 1993, Les Règles du jeu. L'action collective et la régulation sociale, Paris, Armand Colin.

ROCHER Guy, 1989, «Le droit et la sociologie du droit chez Talcott Parsons », Sociologie et Sociétés, 2I-I, p. 143-163.

SALLÉ Loïc, 200I, «Le traitement du dopage par les médias, l'exemple du magazine Sport et Vie», Revue européenne de management du sport, 6, p. I57-192.

-, 2004, Le Gouvernement du dopage en France. Entre pouvoirs publics, acteurs sportifs et médecins. La production de la loi de 1999 comme illustration, Thèse pour le doctorat de Staps, université de Rouen.

-, LESTRELIN Ludovic, BASSON Jean-Charles, 2006, «Tour de France cycliste 1998 et régulation du dopage sportif: reconfiguration des rapports de force», Revue STAPS, 73-3, p. 9-23.

SIMON Gérald, 1990, Puissance sportive et ordre juridique étatique. Contribution à l'étude des relations entre la puissance publique et les institutions privées, Paris, LGDJ.

-, 200 la, «Existe-t-il un ordre juridique du sport?», Droits. Revue française de théorie juridique, 33, p.97-106. 
-, 200 lb, «L'État, le mouvement sportif et le marché: qui fait la loi?», in Jean-Charles Basson (dir.), Sport et ordre public, Paris, La Documentation française-IHESI, p. 37-46.

SLIMANI Hassen, 20I0, «Du jugement social au jugement pénal. L'autonomie du football professionnel français entre arbitraires et arbitrages », Droit et Société, 76, p. 667-69l.

SOULÉ Bastien, BOUHAOUALA Malek, 2008, «Analyse systémique et stratégique du dopage sportif: le cas du cyclisme professionnel », Revue Suisse de sociologie, 34-3, p. 575-592.

STEWART Bob, SMITH Aaron C.T., 2008, «Drug Use in Sport. Implications for Public Policy», Journal of Sport \& Social Issues, 32-3, p. 278-298.

STOKVIS Ruud, 2003, «Moral Entrepreneurship and Doping Cultures in Sport», ASSR Working Paper, Amsterdam School for Social Science Research, [s.p.].

TRABAL Patrick, 2007, «Agir contre le dopage. Les contraintes d'une action publique visant à ajuster les dispositifs à des pratiques insaisissables », communication au colloque Les Approches pragmatiques de l'action publique, Facultés universitaires Saint Louis, Bruxelles.

-, BUISINE Sébastien, DEMESLAY Julie, DEFRANCE Jacques, LE NOÉ Olivier, VERLY Mathieu, 2010, Les Dispositifs antidopage à l'épreuve de la critique, Rapport de recherche pour la MILDT et I'INSERM.

VOY Robert, 1991, Drugs, sport and politics, Champaign, Leisure Press.

WADDINGTON Ivan, 200I, «Doping in sport: Some issues for medical practitioner», Paper presented at the Leiden International Medical Students Congress, University of Leiden Medical School, Leiden, The Netherlands, [s.p.].

WASER Anne-Marie, 2000, «De la règle du jeu au jeu avec la règle», in Françoise Siri (dir.), La Fièvre du dopage, Paris, Autrement, p. 187-198. 
